\title{
EL NUEVO PLAN DE AHORRO DEL GOBIERNO PARA LA GESTIÓN DE LA SALUD LABORAL ¿DONDE ESTÁ LA SALUD LABORAL DE LAS MUJERES CON LA REFORMA DE LAS MUTUAS DE 2014?
}

\author{
Ma Ángeles Romero Coronado ${ }^{1}$ \\ Universidad de Zaragoza
}

http://dx.doi.org/10.5209/rev_NOMA.2014.v43.n3.49280

\begin{abstract}
Resumen.- El mercado laboral es un mundo cambiante y acelerado que viene reflejando las necesidades sociales más básicas. Desde la entrada de la mujer en el mercado laboral en la revolución industrial hasta nuestros días, se viene reivindicando una plena incorporación en todos los aspectos laborales y entre ellos, la salud laboral. La necesaria adaptación al género de la salud laboral ha venido siendo objetivo de las políticas públicas, pero no se ha traducido en una verdadera aplicación. El actual proceso de crisis económica ha llevado a reformar de la gestión de las MUTUAS de accidentes de trabajo y enfermedades profesionales, entidades de vital importancia en el sistema nacional. Esta reforma brinda más libertades a las MUTUAS y hace más difícil la aplicación de la perspectiva de género al reconocimiento de incapacidades temporales y enfermedades profesionales de las mujeres.
\end{abstract}

Palabras clave.- salud laboral, género, poderes públicos, riesgos laborales, enfermedades profesionales e igualdad.

THE GOVERNMENT SAVINGS PLAN NEW FOR OCCUPATIONAL HEALTH MANAGEMENT. ¿WHERE IS THE WORKING WOMEN'S HEALTH REFORM WITH MUTUAS OF 2014?

\begin{abstract}
Labor market it's a changing and accelerated world which reflects the basic social needs. Since the incorporation of women into de labor market in the industrial revolution up to the present time, it's been reclaiming a fully incorporation of all the labor aspects, among including the occupational health for both men and women. The necessity adaption to gender of the occupational health has come to be objective of public policy, but has not been translated into a real application. The ongoing economic crisis has led a new reform of the management MUTUAS of occupational accidents and diseases, vital institutions in the national system. This reform gives more freedom to the MUTUAS and makes it more difficult to apply a gender perspective to the recognition of temporary disabilities and diseases of women.
\end{abstract}

Keywords.- occupational health, gender, government, workplace hazards, occupational diseases and equality.

\footnotetext{
1 Graduada Social; Técnico Superior en Prevención de Riesgos Laborales y Doctorando en Derecho del Trabajo por la Universidad de Zaragoza. Email: marianromerocoronado@outlook.es
} 


\section{Consideraciones generales: las diversas circunstancias laborales y privadas de mujeres y hombres}

Sin lugar a dudas la incorporación de la mujer al mercado laboral se considera como uno de los fenómenos sociodemográficos más importantes del siglo $\mathrm{XX}$, aunque las desigualdades de género en el trabajo son aún acentuadas. $Y$ estas diferencias de género en las condiciones de trabajo tienen una gran repercusión sobre las diferencias de género en cuanto a la salud laboral.

La salud de las mujeres y de los hombres es diferente y es desigual; desigual porque hay otros factores que influyen en la salud de las personas y diferente, porque hay factores biológicos, genéticos, hereditarios, fisiológicos, que se manifiestan de forma diferente en la salud y en los riesgos de enfermedad.

El debate sobre mujer y salud desde la óptica de lo laboral es especialmente relevante dadas las condiciones particulares del empleo femenino actual, las cuales determinan riesgos específicos, a causa de la forma en que se desarrollan sus distintas actividades laborales y los sectores en los que se encajona la fuerza femenina.

Así, en primer lugar, existen diferencias específicas del trabajo de la mujer debido a la forma en que se ha producido su incorporación al mercado de trabajo. Fruto de la existencia de la división sexual del trabajo, tanto en los diferentes sectores de actividad, como en las tareas que se realizan en un mismo sector, según datos de la Encuesta de Población Activa ${ }^{2}$, el trabajo de la mujer se distribuye de forma irregular entre los diversos sectores. La mayoría de las trabajadoras se sitúan en el sector servicios, siendo escasa su participación en el sector industrial. Por otra parte se identifican actividades fuertemente feminizadas: personal del servicio del hogar familiar, actividades de servicios de cuidado personales, actividades sanitarias, veterinarias, servicios sociales, educación, industria textil y confección y comercio al por menor. Actividades que se han entendido propias de las mujeres por ser una prolongación externa de las actividades que venían desempeñando las mujeres en el ámbito doméstico.

Conforme a tales ocupaciones, el trabajo de la mujer presenta, por regla general, unos perfiles propios y diferentes al trabajo desarrollado por los hombres. Como características comunes al trabajo realizado por las mujeres en las ocupaciones mencionadas pueden citarse: la monotonía, falta de creatividad, exigencia de precisión y agilidad con mantenimiento de posturas forzadas e incómodas, ritmo excesivo, escasa cualificación, escasa toma de decisiones y la doble jornada laboral o también conocida como doble presencia ${ }^{3}$.

\footnotetext{
${ }^{2}$ Nota de prensa de 24 de julio de 2014 del Instituto Nacional de Estadística. Encuesta de Población Activa (EPA) 2o trimestre de 2014. Recuperado el 24/08/2014 en: http://www.ine.es/daco/daco42/daco4211/epa0214.pdf

${ }^{3}$ Se define la doble presencia como la situación que se origina cuando recae sobre una misma persona la necesidad de responder a las demandas del trabajo doméstico-familiar y las demandas del trabajo asalariado. La doble presencia está determinada por el aumento de las cargas de trabajo, así como por la dificultad para responder a los requerimientos del trabajo asalariado y doméstico cuando aparecen de manera simultánea. La doble presencia es un riesgo laboral para la seguridad y salud de las personas, siendo asociada a peores indicadores de salud mental, vitalidad y estrés.
} 
Los riesgos laborales asociados a las anteriores condiciones de trabajo son: a) Riesgos Físicos, químicos, biológicos y ergonómicos: derivados del doble uso tanto en el hogar como en el trabajo de productos químicos, la adopción de posturas dolorosas, mantenimiento prolongado de las mismas, movimientos repetitivos que provocan alteraciones muscoloesqueléticas muy dispersas y localizadas en la espalda y miembros superiores así como alteraciones circulatorias en miembros inferiores. B) Riesgos Psicosociales en relación a los trabajos monótonos que requieren escasa cualificación y autonomía y que pueden dar lugar a cuadros de estrés capaces de inducir una mayor tensión muscular. $Y$ en los puestos de trabajo que implican atención a terceros una excesiva carga emocional.

Se constata igualmente que las mujeres están menos expuestas a factores del entorno físico como en ruido, vibraciones, temperaturas extremas o la manipulación de cargas pesadas, circunstancias todas estas que suelen darse en los trabajos históricamente ocupados por hombres. Es una evidencia, por tanto, que existen riesgos que por efecto de la segregación sexual del mercado de trabajo inciden más en las trabajadoras que en los trabajadores.

Al mismo tiempo y por el tipo de factores de riesgo a los que se expone la mujer en los sectores feminizados, los posibles efectos sobre la salud de la mujer trabajadora no son visibles de inmediato ( o no tan evidentes) por consistir en una continuada y progresiva pérdida de salud, a diferencia de las ocupaciones masculinizadas en las que la forma típica de perder la salud deviene de forma súbita e inmediata en forma de accidente de trabajo (los datos de accidentes de trabajo reflejan que estos son tres veces mayor en los hombres que en las mujeres) ${ }^{4}$.

Por otra parte, la forma de incorporación de la mujer al mercado de trabajo ha venido atravesada por otras diferencias de género que influyen en su estado de salud y que tienen que ver con el modelo social tradicional de división del trabajo. La todavía doble presencia de la mujer en el trabajo asalariado o visible y en el trabajo doméstico o invisible, produce una carga de trabajo excesiva, que aumenta el tiempo de trabajo y crea problemas de conciliación de la vida laboral y familiar, pues hombres y mujeres se sitúan de forma diferente frente a tales responsabilidades. A diferencia de roles sociales entre hombres y mujeres hace que la incompatibilidad horaria entre jornada laboral y doméstica se viva e interprete de distinta forma y genere más conflictos para las mujeres, originándose una serie de riesgos psicosociales que indicen de diversa forma en la salud y calidad de vida de ambos sexos.

Relacionado también con la forma de incorporarse al mercado de trabajo, en condiciones generalmente precarias, con miedo a la pérdida del puesto de trabajo, normalmente temporal, fácilmente sustituible, mal retribuido y de escasa cualificación, se ha producido una mayor incidencia en la mujer trabajadora del acoso sexual, demostrándose que

(2012) BRUNEL, Susana. LÓPEZ, Montse y MORENO, Neus. 2a Ed, Guía Sindical. Mujeres, trabajos y salud, Instituto Sindical de Trabajo, Ambiente y Salud (ISTAS), Madrid, pp.25

${ }^{4}$ (2010) DE VICENTE ABAD, Má Ángeles. ZIMMERMANN VERDEJO, Marta. DE LA ORDEN RIVERA, María Victoria y DÍAZ ARABURU, Clara Isabel. Acercamiento a la siniestralidad laboral desde una perspectiva de género, Instituto Nacional de Seguridad e Higiene en el Trabajo (Observatorio Estatal de Condiciones de Trabajo), Madrid, pp.47 
existen grupos específicos de mujeres especialmente vulnerables al mismo (mujeres de minorías étnicas o raciales), tal como demuestran las cifras.

En el caso de las mujeres inmigrantes o pertenecientes a minorías étnicas, la riqueza de su diversidad adquiere más matices, pero la diferencia se vuelve contra ellas en la mayoría de las ocasiones, pues la doble condición de inmigrante y mujer las hace aún más vulnerables.

En segundo lugar, cuando se analiza el trabajo de la mujer debe advertirse diferencias de sexo, de tipo biológico (hormonales, genéticas, anatómicas), que implican en algunos casos un diverso grado de sensibilidad o respuesta ante un riesgo laboral, según el sexo del individuo. Tal es el caso, entre otros de: 1. Mayor sensibilidad de la mujer en relación a la dermatitis de contacto producidas por sustancias químicas. 2. Diferencias entre $\mathrm{M} / \mathrm{H}$ por las alteraciones provocadas por el estrés. 3. Mayor predisposición de la mujer a sufrir el síndrome del túnel carpiano.4. Mayor sensibilidad química múltiple de la mujer (y por tanto mayor probabilidad de sufrir los síntomas del síndrome del edificio enfermo). 5. En relación a los pesticidas y plaguicidas, umbrales de toxicidad más bajos en mujeres que en hombres (relacionadas con trastornos en el ciclo mestrual). 6. Diversa incidencia sobre ambos sexos de los riesgos laborales derivados de la exposición a agentes químicos, físicos o biológicos capaces de ejercer efectos mutagénicos o de toxicidad para la procreación, tanto en los aspectos referidos a la fertilidad como en el desarrollo de la descendencia. 7. La maternidad de la mujer trabajadora como estado biológico que sitúa a la mujer en una situación de especial vulnerabilidad o sensibilidad en relación a determinados riesgos laborales.

Los anteriores datos reflejan una vez más la diversidad de la población trabajadora (esta vez por razón de sexo y género) y con ello que hombres y mujeres puedan vivir de forma diferente siendo diferentes sus respectivos problemas de salud.

\section{Conceptos fundamentales: sexo y género}

Las diferencias entre el trabajo de los $\mathrm{H}$ y $\mathrm{M}$ que se acaban se subrayar derivan fundamentalmente de procesos históricos, sociales y culturales, más que biológicos, que también. Por eso resulta necesario discernir a qué nos referimos con la perspectiva de género aplicada a la salud laboral de las mujeres.

El género, debe ser entendido como una construcción cultural y social en continuo cambio, que asigna tributos y funciones diferentes a hombres y mujeres, y que puede establecer desigualdades en materia de salud, o marcar diferencias innecesarias, evitables e injustas.

La categoría de género, que surge a finales de los años 60 , fue propuesta para afirmar la cualidad fundamentalmente social de las distinciones basadas en el sexo. La misma denota el rechazo al determinismo biológico, implícito en el uso del término sexo.

Así pues, la construcción del género desborda el concepto sexo. De manera que el término sexo se deriva de las características biológicamente determinadas, relativamente invariables del hombre y la mujer, mientras que género se utiliza para señalar las características socialmente construidas que constituyen la definición de los masculino y lo 
femenino en distintas culturas y podría entenderse como la red de rasgos de personalidad, actitudes, sentimientos, valores y conductas que diferencian a los hombres de las mujeres.

A lo largo de la historia la posición de las mujeres en la estructura social se ha asumido mecánicamente como un fenómeno dado, inherente a la naturaleza humana. En este contexto, hasta épocas recientes, el discurso intelectual más elaborado justificaba de manera explícita el sometimiento de las mujeres, remitiéndose a unos principios normativos que no se cuestionaban y que supuestamente derivaban del mandato divino o simplemente estaban inscritos en la naturaleza de las cosas.

En este contexto la teoría femenina, desde sus inicios, ha insistido en afirmar que la posición en la estructura social que se ha asignado a las mujeres es contingente y que, por tanto, no se desprende automáticamente de las diferencias anatómicas o fisiológicas, ni deriva de esencias metafísicas o de la naturaleza de las cosas. Por lo tanto, las racionalizaciones que justificaban la desigualdad de las mujeres deben contemplarse simplemente como construcciones ideológicas segregadas por una sociedad en la que las relaciones de poder son desiguales.

El análisis de género puede utilizarse científica o políticamente para combatir la actitud natural, esto es, la consideración de los patrones de género como una realidad ajena a la intervención humana, irrenunciable e indiscutible. Dicho de otra manera, cuando se contempla la sociedad desde la perspectiva de género puede advertirse que muchas de las pautas socioculturales que se asumen como naturales, objetivas o neutras, están determinadas en gran medida por las relaciones de poder desigualdades entre hombres y mujeres.

Así pues, como se afirmaba, la construcción del género desborda el concepto sexo. De ahí que el enfoque de género aplicado al análisis de la salud de la mujer, debe dirigir la atención hacia las relaciones entre la biología y el medio social. Este enfoque señala diferencias entre hombres y mujeres de acuerdo con las siguientes dimensiones: necesidades especiales de atención. Riesgos específicos ligados a actividades definidas como masculinas o femeninas. Percepciones de enfermedad. Conductas de búsqueda de atención. Grado de acceso y de control ejercido por las personas sobre los recursos básicos para la protección de la salud, etc.

\section{La perspectiva de género en salud pública y salud laboral en el ámbito de la Unión Europea.}

Las políticas de salud pública en el ámbito de la UE han abordado paulatinamente la dimensión de género en los últimos años. Sin embargo, el enfoque aplicado ha consistido en primar las diferencias biológicas y los comportamientos individuales.

El trabajo, ya sea remunerado o no, no se incluye en las política de salud pública cuando éstas tratan la dimensión de género de la salud. $Y$ es que ignoran en gran medida las relaciones sociales entre los sexos, reconociendo al mismo tiempo que los enfoques tradicionales de salud han prestado poca atención a los problemas específicos de las mujeres. 
A nivel europeo, la salud pública se ha considerado explícitamente como una competencia comunitaria a partir del Tatado de Maastricht de 1992, en el artículo 129 del Tratado, pero la UE se limita a desempeñar un papel complementario, no ejerce actividad reguladora.

El único documento que versa sobre la salud de las mujeres es el informe sobre el estado de salud de las mujeres, adoptado por la Comisión en 1997, sin embargo, no se ha puesto en marcha ningún programa específico.

El Tratado de Amsterdam de 1999, amplía las competencias comunitarias en esta materia. El nuevo artículo 152 que sustituye al 129, mantiene la preeminencia de las políticas nacionales. De igual manera el Tratado de Lisboa de 2007 apremia a los Estados miembros a prevenir y combatir la discriminación dentro y fuera del mercado de trabajo.

Por otro lado, las Estrategias comunitarias de salud y seguridad en el trabajo (20022006 y 2007-2012) han insistido en la necesidad de integrar la dimensión de la igualdad entre mujeres y hombres en la evaluación de riesgos laborales, las medidas preventivas y los mecanismos de indemnización, de forma que se tengan en cuenta las particularidades de las mujeres por lo que respecta a la salud y seguridad en el trabajo.

Lo último que se conoce en relación a las Estrategias comunitarias en seguridad y salud, es el comunicado de prensa de fecha 6 de junio de 2014, mediante el que la Comisión Europea pone en conocimiento de todos que actualmente se encuentra en plena tarea de ensamblaje de una nueva estrategia comunitaria para el fomento de la seguridad y salud laboral que abarcaría los años 2014-2020. Mediante esta nueva estrategia se establecerán los objetivos para Europa en materia de seguridad y salud laboral del período 2014-2020.

Se especifica en el comunicado ${ }^{5}$ que la Estrategia 2014-2020 determinará los principales retos y objetivos estratégicos en el ámbito de la salud y la seguridad en el trabajo y presenta las acciones clave y los instrumentos para alcanzarlos. El objetivo de este nuevo marco será garantizar que la UE continúe desempeñando un papel de liderazgo en el fomento de unos estándares elevados en las condiciones de trabajo tanto en Europa como a nivel internacional, en consonancia con la Estrategia Europa 2020. Se prevé una futura revisión de la estrategia para el año 2016, con el fin de hacer balance de su aplicación y para tener en cuenta los resultados de la evaluación global de la legislación de la UE sobre salud y seguridad profesionales que se está llevando a cabo. Pero el documento no estará disponible hasta finales de 2015.

\section{La Ley Orgánica $3 / 2007$, de Igualdad efectiva de mujeres y hombres y la incorporación de la perspectiva de género en el ámbito de la salud pública y de la salud laboral.}

La perspectiva de género en nuestro país, viene determinada principalmente con la LOIMH, aunque se encuentre el término género implícito en otras normas nacionales.

\footnotetext{
${ }^{5}$ Comunicado de prensa de la Comisión Europea de 6 de junio de 2014. Bruselas. Recuperado el 16/09/2014 en: http://europa.eu/rapid/press-release_IP-14-641_es.htm
} 
El artículo 27 LOIMH dispone la integración del principio de igualdad en las políticas de salud pública, indicando que las Administraciones Públicas se centrarán en evitar que por razón de diferencias biológicas o por estereotipos sociales asociados se produzcan discriminaciones entre uno y otro sexo, con una política específica de promoción de la salud de la mujer, así como la prevención de su discriminación en el marco de la educación sanitaria y la investigación científica y, en particular, en relación a la salud laboral, el acoso sexual y acoso por razón de género.

Consecuencia de este precepto resultó ser la modificación del artículo 21 de la Ley General de Sanidad, donde se regula la actuación sanitaria en el ámbito de la salud laboral, para integrar específicamente, $y$, en todo caso, la perspectiva de género. Precepto éste modificado por el artículo 33 de la Ley 33/2011 de 4 de octubre, General de Salud Pública, de la «actuación sanitaria en el ámbito laboral».

Por otra parte, la promulgación de la LOIMH supondrá también la incorporación al artículo 5 de la Ley 31/1995, de 8 de noviembre de Prevención de Riesgos Laborales (LPRL), de un nuevo apartado 4, donde se señala que: «Las Administraciones Públicas promoverán la efectividad del principio de igualdad entre mujeres y hombres, considerando las variables relacionadas con el sexo tanto en el sistema de recogida y tratamiento de datos como en el estudio e investigaciones generales en materia de prevención de riesgos laborales, con el objetivo de detectar y prevenir posibles situaciones en la que los daños derivados del trabajo puedan aparecer vinculados con el sexo de los trabajadores».

\section{Consideraciones sobre el infra- reconocimiento de la salud laboral de las mujeres}

El acoso sexual y acoso por razón de sexo se consideran "riesgos de carácter psico-social" y genéricamente se trata de toda situación susceptible de producir un daño a la salud psíquica y al bienestar de las personas en virtud de la interacción entre el trabajo, su ambiente y las condiciones de su organización, por una parte, y, por otra, las capacidades de la persona trabajadora, sus necesidades, su cultura y experiencia. Se definen por la LOIMH como: “...constituye acoso sexual cualquier comportamiento, verbal o físico, de naturaleza sexual que tenga el propósito o produzca el efecto de atentar contra la dignidad de una persona, en particular cuando se crea un entorno intimidatorio, degradante $u$ ofensivo. Constituye acoso por razón de sexo cualquier comportamiento realizado en función del sexo de una persona, con el propósito o el efecto de atentar contra su dignidad y de crear un entorno intimidatorio, degradante u ofensivo."

Estos tipos de acoso laboral afectan más a las mujeres que a los hombres, bien, por el simple hecho de ser mujeres (acoso sexual) o bien, por el ejercicio de los derechos relacionados con la conciliación de la vida familiar, personal y laboral (acoso por razón de sexo), provocando alteraciones en la salud de las mismas como son los trastornos ansiososdepresivos. Añádase a lo dicho un nuevo tipo de acoso ambiental contra las mujeres que se 
identifica con la violencia que muchas mujeres sentimos en el trabajo por el mero hecho de incorporarnos a un mundo laboral pensado en masculino, donde en muchísimas ocasiones y de la manera más evidente y también de la más sutil, nos sentimos excluidas al no ser capaces de encontrar una manera de ser mujeres trabajadoras libres. De hecho se intuye que existe una directa relación entre este tipo de acoso y el dolor crónico de muchas enfermedades nuevas e invisibles que padecemos, como son la fibromialgia, la fatiga crónica u otros problemas endocrinos típicamente femeninos.

En la práctica se constata que muchas de las enfermedades que padecen las mujeres, consecuencia del entorno laboral en el que desarrollan su actividad, no son consideradas enfermedades del trabajo, sino que se derivan al Sistema Público de Salud. Ocurre con los supuestos de riesgos durante el embarazo o durante la lactancia natural, o con muchas situaciones de estrés o trastornos ansioso-depresivos que derivan de supuestos de acoso 0 conflictividad en el ámbito laboral. Las consecuencias de esta derivación son, por un lado, que se trasladan los costes de las enfermedades profesionales al sistema público de salud y, por otro lado, que las prestaciones de seguridad social que reciben las mujeres son inferiores a la que correspondería de considerarse una enfermedad profesional o enfermedad derivada del trabajo. Interviene en esta situación el hecho de que más del $90 \%$ las prestaciones derivadas de contingencias profesionales son gestionadas por las MUTUAS de Accidentes de trabajo, lo que ha llevado a la propia Administración a desarrollar sistemas de revisión: primero a través de la Resolución de la Secretaría de Estado de la Seguridad Social de 19 de septiembre de 2007, donde se desarrollaron una serie de criterios o pautas dirigidas a la adecuada calificación de las contingencias por parte del INSS, respecto de los expedientes tramitados por las MUTUAS en materia de Incapacidad Temporal y por muerte y supervivencia resueltos sin considerar como enfermedad profesional. Pasando por el RD 1430/2009, de 11 de septiembre, por el que se desarrolló reglamentariamente la Ley 40/2007, de 4 de diciembre, de medidas en materia de Seguridad Social, en relación con la prestación de incapacidad temporal, en cuyo artículo 4 se desarrolla el procedimiento administrativo de revisión de las altas médicas expedidas en los procesos de incapacidad temporal, frente a las altas médicas emitidas por las MUTUAS. Hasta la actualidad, con un plan integral de medidas de reforma de las MUTUAS colaboradoras de la Seguridad Social y de la gestión de la Incapacidad Temporal, 18 de julio de 2014.

Por tanto, la comunicación entre el sistema público de salud y los sistemas de salud laboral de las empresas deberían coordinarse para que se evitaran este tipo de disfunciones, situación difícil con el reciente cambio normativo.

\section{De la Coordinación entre las políticas de salud laboral y las políticas de salud pública}

La salud laboral no es sino manifestación del principio general de salud pública, que, en consecuencia, comparte con éste el objetivo de conseguir una mejora constante del bienestar 
general, del que la salud laboral es una parte fundamental. Por tanto, la salud laboral no es, sino, una especificación de la salud globalmente considerada y dependiente de esta.

Esta interdependencia ya se puso de manifiesto en la Ley General de Sanidad de 1986; con posterioridad en varios preceptos de la LPRL y recientemente se ha intensificado en el Ley 33/2011 de 4 de octubre, General de Salud Pública (LGSP).

$Y$ aunque la salud laboral tiene un tratamiento diferenciado de la asistencia sanitaria universal y no contributiva, en tanto que la asistencia sanitaria por enfermedad profesional y accidentes de trabajo se fundamenta en el principio de responsabilidad empresarial, no es menos cierto que el objetivo de alcanzar la vigilancia integral de la salud pública, que proclama el preámbulo de la LGSP, requiere abarcar todos los condicionamientos de la salud, también los que se producen en el ámbito laboral. No obstante se reconoce que a pesar de que se disponen de avanzados indicadores sobre la enfermedad, sobre la asistencia sanitaria y sobre algunas de las conductas relacionadas con la salud, tal información no está integrada con la procedente de otros ámbitos sociales, ambientales o de otro carácter, esenciales para valorar la evolución de la salud pública y las políticas relacionadas con ellas.

Por eso resulta fundamental establecer acciones de coordinación y colaboración de las Administraciones Públicas en materia de salud pública, tal como se manifiesta en el preámbulo de la LGSP 33/2011. Dicha coordinación se pretende con políticas de carácter sanitario, ambiental, económico, alimentario, de promoción de la actividad física y también con las políticas laborales. De ahí que entre los objetivos específicos que proclama la LGSP se encuentra la de establecer «acciones en materia de salud laboral para conseguir la mejor prevención de riegos en el ámbito laboral, y en coherencia con lo dispuesto en la LPRL, y complementándola se facilita la promoción de la salud en ese mismo ámbito». La promoción y protección de la salud laboral, continua el preámbulo de la ley, así como la prevención de los factores de riesgo en este ámbito, deben ser contempladas en la cartera de servicios de la salud pública.

La necesaria coordinación en salud laboral entre el sistema público y las actividades sanitarias que se realizan en las empresas es uno de los objetivos de la política de seguridad y salud laboral, contemplados en el Capítulo II de la LPRL, y concretados en el artículo 10 LPRL.

El artículo 10 de la LPRL, encomienda las competencias en materia sanitaria referentes a la salud laboral a las AAPP dependientes del Ministerio de Sanidad y Consumo (hoy Ministerio de Sanidad, Servicios Sociales e Igualdad). En concreto determina que tales actuaciones se llevarán a cabo en los términos establecidos en el Capítulo IV, del Título I de la Ley 14/1986, de 25 de abril, General de Sanidad, y disposiciones dictadas para su desarrollo, (hoy artículo 33 de la LGSP).

Sin embargo, y tras semejantes apuestas por la salud laboral desde los poderes públicos, en fecha 18 de julio de 2014 el Consejo de Ministros aprobó, a propuesta de la ministra de Empleo y Seguridad Social, un plan integral de medidas de reforma de las MUTUAS colaboradoras de la Seguridad Social y de la gestión de la Incapacidad Temporal, en el marco de lo establecido en el Programa Nacional de Reformas. 
Este plan se compone de un Proyecto de Ley por el que se modifica el texto refundido de la Ley General de la Seguridad Social en relación con el régimen jurídico de las MUTUAS de Accidentes de Trabajo y Enfermedades Profesionales de la Seguridad Social y el Real Decreto que regula determinados aspectos de la gestión y control de los procesos de Incapacidad Temporal (IT) en el primer año de duración.

El Gobierno actual, con este proyecto pretende potenciar el negocio de las MUTUAS y aplicar nuevos recortes a los derechos de los trabajadores, este cambio legal permitirá a las MUTUAS de accidentes de trabajo y enfermedades profesionales involucrarse en los procesos de incapacidad temporal por contingencias comunes desde el primer día de la baja.

El proyecto de Ley es un nuevo ataque contra los derechos laborales y sociales de los trabajadores y trabajadoras, es una reforma ideológica, basada en criterios meramente economicistas que atenta gravemente los derechos de los pacientes, los trabajadores/as y los profesionales del Sistema Nacional de Salud (SNS).

Tanto el Real Decreto 625/2014 de 18 de julio, de gestión de la incapacidad temporal en el primer año de duración y el proyecto de Ley de MUTUAS, por el que se concede a estas entidades una mayor capacidad de intervención en las bajas médicas por enfermedad común, cuentan con el objetivo de reducir el absentismo laboral injustificado.

Empleo estima que se conseguirá un ahorro cercano a los 300 millones de euros en la gestión de la incapacidad temporal con la mayor intervención de las MUTUAS en estos procesos. Y es que en concreto, las MUTUAS podrán realizar el seguimiento de la prestación de incapacidad temporal derivada de enfermedad común desde el primer día de la baja (hasta ahora sólo podían intervenir a partir del décimosexto día) y formular, como ocurre actualmente, una propuesta de alta médica "motivada".

Para ello, se sustituye el actual sistema de expedición de los partes médicos de confirmación -de periodicidad semanal- por otro basado en la distinción de cuatro grupos de procesos (muy cortos, cortos, largos o muy largos), con distinta periodicidad en la expedición de los indicados partes.

A tal fin, la ministra ha explicado que se tomará como referencia una tabla orientativa de duración estimada de la IT del trabajador en función de su patología, edad y su ocupación real. Esta información no es vinculante, sino de naturaleza informativa.

En concreto, se establece que, en los procesos en los que se estimen cinco días de baja, el parte de baja y el de alta se podrán expedir en la misma visita médica. Si la duración de la IT se estima en entre cinco y 30 días, el parte de confirmación de la baja deberá expedirse cada dos semanas; si el proceso está previsto que dure entre 31 y 60 días, el parte de confirmación se dará cada 28 días, y en enfermedades de larga duración, de más de 61 días, la confirmación de la baja se expedirá cada 35 días.

\section{Valoración Conclusiva: actuaciones concretas a desarrollar por el Sistema Público de Salud para el reporte en la salud laboral de género}


Antes de pasar al desglose de las recomendaciones a los poderes públicos, es necesario reflexionar sobre la nueva normativa en salud laboral.

Lo que actualmente tenemos encima de la mesa es que si con la regulación anterior las MUTUAS ya infravaloraban los riesgos derivados de contingencias profesionales de las mujeres y las declaraciones de IT y Enfermedad profesional femeninas venían encuadrándose en contingencias comunes por el espaciamiento en el tiempo de aparición de las mismas y lo paulatinas que son. Ahora que las MUTUAS tienen más competencias y libertades en la actuación, olvidémonos de una gestión coordinada entre poder público y privado que reporte positivamente en la salud laboral, y olvidémonos de una óptica del género.

Recomendaciones a los poderes públicos:

1. Impulsar la incorporación de la perspectiva de género en la salud en general y en la salud laboral en particular. Principalmente esta última a través de la prevención de riesgos laborales, incorporando en todos los procesos de evaluación de riesgos y en las medidas preventivas, de forma que se tenga en cuenta las particularidades de las mujeres en lo que respecto a la seguridad y salud en el trabajo.

Indicadores de salud laboral que deberían contemplarse en todas las evaluaciones de riesgos laborales desde una perspectiva de género:

a) Datos sobre las condiciones laborales (sectores profesionales, jornada laboral, antigüedad), las características del trabajo (contacto con las personas, carácter repetitivo, autonomía) y las características del empleo (tipo de contrato).

b) Datos sobre la exposición a factores de riesgo.

c) Datos sobre el estado de salud (accidentes laborales, enfermedades profesionales, absentismo, otras patologías), ampliadas eventualmente mediante una noción amplia de calidad en el trabajo. Realizar encuestas de calidad en el trabajo.

d) Datos sobre la organización de la prevención (acceso a la vigilancia de la salud, cobertura por los servicios de prevención, representación de los trabajadores en el ámbito de la salud laboral, formación específica en materia de salud laboral).

e) Datos sobre las personas afectadas (edad, sexo, nacionalidad, nivel de estudios, nivel salarial, situación laboral anterior, antigüedad en la empresa, situación familiar).

2. Desarrollar acciones de sensibilización y formación en prevención de riesgos laborales desde la perspectiva de género, dirigidos especialmente a los trabajadores y empresarios.

3. Elaborar trabajos, documentos y materiales relativos a la prevención de riesgos laborales relacionados con la seguridad y salud de la mujer en el trabajo.

4. Impulsar la realización de estudios estadísticos con datos desagregados por sexos para comprobar el infra-reconocimiento de las enfermedades profesionales en las mujeres y los índices de siniestralidad. Se deberían recoger datos sobre las siguientes cuestiones:

a) Evaluación de los accidentes de trabajo con baja en jornada de trabajo, por sector económico, gravedad y género. 
b) Partes de declaración de enfermedad profesional según género y grupo de enfermedad (agentes químicos, de la piel, agentes físicos, inhalación de sustancias, etc..).

c) Indicadores de incidencia de declaración de enfermedad profesional según edad y género.

d) Trabajadores/as que han sufrido algún tipo de conducta de acoso según la rama de actividad (frecuencia diaria, semanal, mensual).

e) Partes de accidente de trabajo con baja según el grado de la lesión y la actividad económica.

El tratamiento de los datos reflejan la poca importancia que las políticas públicas otorgan a la salud laboral. Los datos desagregados por sexo suelen estar disponibles en los sistemas de información, pero tienen carencias, por lo que no bastan por sí solos para realizar un análisis de género de las situaciones. El sexo como variable autónoma no sirve para explicar gran cosa en el ámbito de la salud laboral, hay que incluir datos como: formas de empleo, tiempo de trabajo, sectores de actividad, tareas desempeñadas. La mayoría de los datos de salud pública no permiten evaluar el impacto de las condiciones de trabajo en la salud. Incluso al abordar específicamente las desigualdades sociales en materia de salud, la relación con las condiciones laborales no suele ser objeto de análisis profundo.

5. Impulsar el desarrollo de las actuaciones de la administración sanitaria en materia de salud laboral, contenidas en el artículo 33 de la LGSP, en especial la referida a la formación en salud laboral de los profesionales sanitarios de los sistemas sanitarios públicos. 


\section{Referencias bibliográficas}

BLANCO A. (1998), "Teorías de la conciencia feminista". En Jagoe, C.; Blanco, A. y Enríquez de Salamanca, C. La mujer en los discursos de género. Textos y contextos en el siglo XIX. Ed. Icaria, Barcelona, pp. 445-472

BRUNEL, S. LÓPEZ, M. y MORENO, N. (2012) Guía Sindical. Mujeres, trabajos y salud, Instituto Sindical de Trabajo, Ambiente y Salud (ISTAS), Madrid

CASTAÑAGA, C. (2012) "Salud Ocupacional: Historia y retos del futuro", Revista Peruana de Medicina Experimental y Salud Pública, 29(2), ISSN 1726-4634, Lima, pp.177-178

DE VICENTE ABAD, M. ZIMMERMANN VERDEJO, M. DE LA ORDEN RIVERA, María V. y DÍAZ ARABURU, Clara I. (2010) Acercamiento a la siniestralidad laboral desde una perspectiva de género, Instituto Nacional de Seguridad e Higiene en el Trabajo (Observatorio Estatal de Condiciones de Trabajo), Madrid

DURÁN Y LALAGUNA, P. (2007) Sobre el género y su tratamiento en las Organizaciones Internacionales, Ediciones Internacionales Universitarias, S.A., Madrid

ESTEBAN, ML. (2006) "El estudio de la salud y el género. Las ventajas de un enfoque antropológico y feminista", Salud Colectiva, (2)1, ISSN 1851-8265, Buenos Aires, pp.9-20

GARCÍA GÓMEZ, M. (2005) "La salud laboral y la salud pública: 10 años de políticas sanitarias de salud laboral", Archivos de Prevención de Riesgos Laborales, 8(3), ISSN 11389672, Barcelona, pp.100-102

PIETILA, H. y VICKERS, J. (1990) Making women matter: the role of the United Nations, Ed. Zeed Books, London

RUÍZ FRUTOS, C. DELCLÓS, J. RONDA, E. GARCíA, Ana M. y BENAVIDES. Fernando G. (2013) Salud Laboral. Conceptos y técnicas para la prevención de riesgos laborales, Ed. Elsevier Masson, Barcelona

VOGEL, L. (2006) La salud de la mujer trabajadora en Europa. Desigualdades no reconocidas, Instituto Sindical de Trabajo, Ambiente y Salud (ISTAS), Madrid

www.boe.es

www.empleo.gob.es

www.eu.vlex.com

www.inmujer.es

www.ilo.org

www.un.org

www.who.int 